\title{
Weakly Nonlocal And Nonlinear Heat Transport In Rigid Solids
}

G. Lebon ${ }^{1}$, D. Jou ${ }^{2}$, J. Casas-Vázquez ${ }^{2}$, W. Muschik ${ }^{3}$

${ }^{1}$ Liège University, Institute of Physics B5, Liège, Belgium

${ }^{2}$ Autonomous University of Barcelona, Department of Statistical Physics, Bellaterra, Catalonia, Spain

${ }^{3}$ Technical University Berlin, Institute for Theoretical Physics, Berlin, Germany

Communicated by A. Bejan, Durham, USA and G. A. Maugin, Paris, France

Registration Number 780

\begin{abstract}
A weakly nonlocal and nonlinear theory of heat conduction in rigid bodies is proposed. The constitutive equations generalize these of Fourier, Maxwell-Cattaneo and Guyer-Krumhansl. The proposed model uses the fundaments and the technique of extended irreversible thermodynamics. The main conclusion is that the presence of nonlocal terms in the transport equation for the heat flux implies a modification of the entropy flux; the latter is no longer given by its classical expression, i.e. the heat flux divided by the temperature, but contains extra contributions which are nonlinear in the heat flux and its gradient. These results arise as compatibility conditions with the second law of thermodynamics. A nonequilibrium temperature depending on the heat flux and generalizing the local equilibrium temperature is also emerging naturally from the formalism.
\end{abstract}

\section{Introduction}

Memory effects are playing an important role in recent researches on heat waves and hyperbolic heat transport [1-3] in solids. The simplest way to take such effects into account is to use the Maxwell-Cattaneo equation

$$
\tau \dot{\mathbf{q}}+\mathbf{q}=-\lambda \nabla T
$$

wherein $\mathbf{q}$ is the heat flux, $T$ the local equilibrium temperature, $\tau$ the relaxation time of the heat flux and $\lambda$ the thermal conductivity, an upper dot stands for derivation with respect to time; the classical Fourier law is recovered in the limit $\tau \rightarrow 0$. When combined with the energy balance equation, expression (1) leads a hyperbolic differential equation for the temperature predicting that thermal waves propagate with a finite velocity. 
This problem is presently a subject of vivid interest and solutions of the hyperbolic temperature equation have been discussed in a wide diversity of situations [1-4].

From the thermodynamic point of view, it turns out that equation (1) requires a modification of the entropy, in such a way that instead of the local equilibrium entropy $s_{e q}(u)$, a generalized entropy depending both on $u$ and $\mathbf{q}$ must be used, it has been shown [5-11] that this generalized entropy will take the form

$$
s(u, \mathbf{q})=s_{e q}(u)-\frac{\tau}{2 \lambda T^{2}} \mathbf{q} \cdot \mathbf{q},
$$

wherein $s$ and $s_{e q}$ are measured per unit volume. In the limit $\tau \rightarrow 0$, this expression reduces to the local equilibrium entropy.

Such a generalized entropy has been the basis of the development of a new thermodynamic theory called Extended Irreversible Thermodynamics (EIT) [e.g. 9]. In this formalism, the thermodynamic fluxes, like the heat flux, the flux of matter and the flux of momentum, i.e. the viscous stress tensor, are elevated to the rank of independent variables, on the same footing as the classical variables, like energy, mass and momentum. In addition, the existence of a nonequilibrium entropy depending on the whole set of variables, and whose rate of production is non-negative is taken for granted. In the framework of EIT, Fourier and Stokes-Newton's laws are generalized in the form of time-rate evolution equations of the Maxwell-Cattaneo type. Such equations are nonlocal in time, as they imply memory effects, but local in space. The analysis of systems subject to important spatial gradients, or characterized by short wavelengths, has fostered the interest in formulating thermohydrodynamic constitutive equations containing nonlocal spatial terms; of particular interest are systems of small dimensions comparable to the mean free path, as microelectronic devices, and ballistic propagation of phonons at low temperature.

Nonlocal effects can be introduced in several ways [2, 3, 12-16]. The simplest one would be, for instance to generalize (2) as

$$
\tau \dot{\mathbf{q}}+\mathbf{q}=-\lambda \nabla \boldsymbol{T}+l_{1}^{2}\left[\nabla^{2} \mathbf{q}+2 \nabla(\nabla \cdot \mathbf{q})\right]
$$

wherein the coefficient $l_{1}$ has the dimension of length.

An equation of this form was derived by Guyer and Krumhansl [17] from the Boltzmann equation for phonons in the relaxation time approximation. This equation is useful to describe the so-called phonon-hydrodynamic regime. More general equations have recently been proposed to describe the ballistic propagation of phonons at high frequencies [18].

In view of (3), a natural question is whether the nonlocal terms imply some modifications in the expressions of the entropy and the entropy flux. We will show that space non-locality does not influence the entropy, but that it contributes to the entropy flux $\mathbf{J}^{s}$. Indeed, instead of the classical result $\mathbf{J}^{s}=T^{-1} \mathbf{q}$, the entropy flux 
will, in presence of non-locality, take the form

$$
\mathbf{J}^{s}=\theta^{-1} \mathbf{q}+l_{1}^{2}\left(\lambda T^{2}\right)^{-1}(\nabla \mathbf{q}) \cdot \mathbf{q}+l_{2}^{2}\left(\lambda T^{2}\right)^{-1}(\nabla \cdot \mathbf{q}) \mathbf{q}+l_{3}^{2}\left(\lambda T^{2}\right)^{-1} \mathbf{q} \cdot(\nabla \mathbf{q})
$$

where $l_{1}, l_{2}, l_{3}$ are phenomenological coefficients, $\theta$ is the temperature, defined by $\theta^{-1}=\partial s / \partial u$ and therefore, depending generally on the heat flux. Expression (4) is revealing as it shows explicitly the relation between the transport equation (3) and the entropy flux: any modification of the transport equation will directly influence the entropy flux and vice-versa.

The question about the form of the entropy flux has been discussed many times in non-equilibrium thermodynamics. Müller [19] proposed that the entropy flux is not just $\mathbf{q} / T$ but that it may have nonclassical contributions, different of course from the classical contribution $-\left(\mu_{i} / T\right) \mathbf{J}_{i}$ related to the transport of matter of component $i, \mu_{i}$ is the chemical potential and $\mathbf{J}_{i}$ the mass flux of component $i$. In Müller's view [24], the entropy flux should be given by $\mathbf{J}=\Lambda^{-1}(T, \dot{T}) \mathbf{q}$ where $\Lambda$ is the so-called coldness which depends not only on the temperature but also on its time derivative. However, this idea was contested by Meixner [20] and was abandoned later on by Müller himself. Other examples of nonclassical expressions of the entropy flux are these proposed by Grad [21] in the framework of the thirteen-moment approximation, namely

$$
\mathbf{J}^{s}=T^{-1} \mathbf{q}-\frac{2}{5 p T} \mathbf{P}^{v} \cdot \mathbf{q}
$$

$\left(\mathbf{P}^{v}\right.$ is the viscous pressure tensor), and the information theoretical expression $[22,23]$

$$
\mathbf{J}^{s}=\gamma_{0} \mathbf{q}+\gamma_{1} \cdot \mathbf{Q}
$$

where $\gamma_{0}$ (a scalar) and $\gamma_{1}$ (a vector) are Lagrange multipliers related to the constraints on the mean value of the internal energy $u$ and the heat flux $\mathbf{q}, \mathbf{Q}$ is the flux of the heat flux, a second order tensor. Since $\gamma_{0}=1 / \theta$, rather than $1 / T$, with $T$ the local equilibrium temperature, expression (6) is more general than (5). The latter may be obtained from the former provided one assumes that $\mathbf{Q}$ is proportional to $\mathbf{P}^{v}$ and that the temperature $\theta$ reduces to the (local) equilibrium value $T$.

Nevertheless, some authors [23] have proposed to keep for $\mathbf{J}^{s}$ the classical form $T^{-1} \mathbf{q}$, by taking this relation as an imposed constraint on the system in an information theoretical approach. Of course, this is in principle possible, but if one ignores the spatial correlations in the entropy flux then they will appear in the expression for the entropy.

In Section 2, we establish a generalized nonlinear transport equation for the heat flux vector $\mathbf{q}$ in undeformable solids; this expression contains as particular cases, the Fourier, Cattaneo and Guyer-Krumhansl equations. Restrictions on the possible forms of the evolution equation are placed by the second law of thermodynamics and are 
described in Section 3; in addition, general expressions of the temperature, entropy and entropy flux compatible with the transport equations and the laws of thermodynamics are derived. To clearly emphasize the correlation between the heat transport equation and the entropy flux, simplified situations are discussed in Section 4 and final conclusions are drawn in Section 5.

\section{A nonlinear and nonlocal heat transport equation}

We consider the problem of unsteady heat conduction in a rigid body. According to Extended Irreversible Thermodynamics [9], the state space is formed by the internal energy, $u$, taken here per unit volume and the heat flux vector q. As a preliminary, it should be stressed that in the present formalism, the gradients of the basic variables $u$ and $\mathbf{q}$ will not be considered as independent variables obeying evolution rate equations. Instead, non-locality is introduced in the constitutive equations; however we will restrict our analysis to a weakly nonlocal theory with constitutive equations involving gradients of the first order with respect to the variables, as it embraces still sufficient generality; it is also our opinion that a more general and formal description would obscure the physical content. For simplicity, we will also address our attention to isotropic systems.

We start with establishing the evolution equations for the basic variables $u$ and $\mathbf{q}$. Concerning the internal energy, its evolution in the course of time and space is governed by the first law of thermodynamics

$$
\dot{u}=-q_{j, i} \delta_{i j}+r
$$

a comma stands for derivation with respect to space, $r$ is the source of energy per unit volume, $\delta_{i j}$ is Kronecker's symbol, Cartesian coordinates as well as Einstein's summation convention on repeated indices will be used throughout the paper. Later on, for simplicity, the source term in (7) will be ignored. By analogy with (7), the evolution equation for $q_{i}$ will be written in the form

$$
\dot{q}_{i}=-\Phi_{i j, j}^{q}+\sigma_{i}^{q}
$$

wherein $\Phi_{i j}^{q}$ is the flux of heat flux (a second-rank tensor), and $\sigma_{i}^{q}$ the corresponding source term (a vectorial quantity). The quantities $\Phi_{i j}^{q}$ as well as $\sigma_{i}^{q}$ will be expressed by means of constitutive equations: it is assumed that $\Phi_{i j}^{q}$ and $\sigma_{i}^{q}$ may depend nonlinearly on $u$ and $q_{i}$ but that they are linear function of their gradients (weak nonlocality). Admissible expressions for $\Phi_{i j}^{q}$ and $\sigma_{i}^{q}$ are therefore

$$
\begin{aligned}
\Phi_{i j}^{q}= & A\left(u, q^{2}\right) \delta_{i j}+B\left(u, q^{2}\right) q_{i} q_{j}-L_{1}\left(u, q^{2}\right) q_{i, j} \\
& -L_{2}\left(u, q^{2}\right) q_{k, k} \delta_{i j}-L_{3}\left(u ; q^{2}\right) q_{j, i}, \\
\sigma_{i}^{q}= & -a\left(u, q^{2}\right) q_{i}-b\left(u, q^{2}\right) u_{, i}+F_{j}\left(u, q_{k}\right) q_{j, i} \\
& +q_{i, j} H_{j}\left(u, q_{k}\right)+G_{i}\left(u, q_{k}\right) q_{j, j},
\end{aligned}
$$

J. Non-Equilib Thermodyn. $\cdot 1998 \cdot$ Vol $23 \cdot$ No. 2 
wherein $q^{2}$ stands for $q_{i} q_{i}$, the minus sign in front of $a, b, L_{1}, L_{2}$, and $L_{3}$ has been introduced for convenience. Substitution of (9) and (10) in the evolution relation (8) leads to

$$
\begin{aligned}
\dot{q}_{i} & =-\frac{\partial A}{\partial u} u_{, i}+B q_{i, j} q_{j}+B q_{i} q_{j, j}+L_{1} q_{i, j j}+\frac{\partial L_{1}}{\partial u} u_{, j} q_{i, j}+L_{2} q_{j, j i}+\frac{\partial L_{2}}{\partial u} u_{, i} q_{k, k} \\
& +L_{3} q_{j, i j}+\frac{\partial L_{3}}{\partial u} u_{, j} q_{j, i}-a q_{i}-b u_{, i}+F_{j} q_{j, i}+q_{i, j} H_{j}+G_{i} q_{j, j} .
\end{aligned}
$$

In expression (11), we have omitted third order terms like $\frac{\partial L_{1}}{\partial q_{k}} q_{k, j} q_{i, j}$ involving the products of $\mathbf{q}$ and the gradients of the variables; in addition, the term $\left(\partial A / \partial q_{j}\right) q_{j, i}$ has been incorporated in the term $F_{j} q_{j, i}$ and therefore does not appear explicitly. To recover earlier familiar results, we introduce the following notation

$$
a=\frac{1}{\tau}, \frac{\partial A}{\partial u}+b=\kappa, \quad L_{i}^{\prime}=\frac{\partial L_{i}}{\partial u} \quad(i=1,2,3),
$$

while $F_{j}, H_{j}$ and $G_{j}$ are supposed to be linear in $q_{j}$ and $u_{, j}$ :

$$
F_{j}=\alpha_{1}(u) q_{j}+\alpha_{2}(u) u_{, j}, H_{j}=\tilde{\beta}_{1}(u) q_{j}+\beta_{2}(u) u_{, j}, G_{j}=\tilde{\gamma}_{1}(u) q_{j}+\gamma_{2}(u) u, j .
$$

Equation (11) reads then:

$$
\begin{aligned}
\dot{q}_{i}= & -\kappa(u) u_{, i}-\frac{1}{\tau(u)} q_{i}+\alpha_{1}(u) q_{j} q_{j, i}+\beta_{1}(u) q_{i, j} q_{j}+\gamma_{1}(u) q_{j, j} q_{i} \\
& +\alpha_{2}(u) u_{, j} q_{j, i}+\beta_{2}(u) q_{i, j} u, j+\gamma_{2}(u) q_{j, j} u_{, i}+L_{1}(u) q_{i, j j} \\
& +L_{2}(u) q_{j, j i}+L_{3}(u) q_{j, i j}+L_{1}^{\prime}(u) u_{, j} q_{i, j} \\
& +L_{2}^{\prime}(u) u_{, i} q_{j, j}+L_{3}^{\prime}(u) u_{, j} q_{j, i}
\end{aligned}
$$

wherein $\beta_{1}(u)$ and $\gamma_{1}(u)$ stand for $\tilde{\beta}_{1}(u)+B(u)$ and $\tilde{\gamma}_{1}(u)+B(u)$ respectively, and the dependence of the various coefficients $\tau, \kappa, L_{1}, \ldots$ with respect to $\mathbf{q}$ has been neglected as they would contribute to third order terms in $q^{3}, q^{2} u_{i}, q^{2} q_{i, j j}, \ldots$ Relation (14) is rather general because it contains as particular cases the laws of Guyer and Krumhansl [17], Cattaneo [24] and Fourier. Indeed by letting in (14) the quantities $\alpha_{i}, \beta_{i}$ and $\gamma_{i}(i=1,2,3 ;)$ tend to zero and assuming that $\tau, L_{1}, L_{2}$ and $L_{3}$ are constant, one recovers a:Guyer-Krumhansl type equation, namely

$$
\dot{q}_{i}=-\kappa(u) u_{, i}-\frac{1}{\tau} q_{i}+L_{1} q_{i, j j}+L_{2} q_{j, j i}+L_{3} q_{j, i j} .
$$

If in addition $L_{1}, L_{2}$ and $L_{3}$ vanish, one rediscovers Cattaneo's equation while in the approximation $\tau=0$ (but $\tau \kappa$ finite), one recovers Fourier's law. 


\section{Restrictions placed by the second law of thermodynamics}

We assume the existence of a non-equilibrium entropy $s$ per unit volume satisfying a balance equation of the form

$$
\dot{s}=-J_{i, i}^{s}+\sigma^{s}\left(\sigma^{s} \geq 0\right)
$$

$J_{i}^{s}$ is the entropy flux and $\sigma^{s}$ the rate of entropy production per unit volume; according to the second law of thermodynamics, $\sigma^{s}$ is a positive definite quantity. In (16), both $s$ and $J_{i}^{s}$ have to be expressed by means of constitutive equations which, in all generality may be written as

$$
s=s\left(u ; q_{j} ; u, j ; q_{j, k}\right), \quad J_{i}^{s}\left(u ; q_{j} ; u, j ; q_{j, k}\right) .
$$

The dependence of $s$ and $J_{i}^{s}$ on the gradients of $u$ and $q_{i}$ is introduced in view of the (weakly) nonlocal description. After substitution of (17) in (16), one obtains for $\sigma^{s}$ the following expression:

$$
\begin{aligned}
\sigma^{s}= & \frac{\partial s}{\partial u} \dot{u}+\frac{\partial s}{\partial q_{i}} \dot{q}_{i}+\frac{\partial J_{i}^{s}}{\partial u} u_{, i}+\frac{\partial J_{i}^{s}}{\partial q_{j}} q_{j, i}+\frac{\partial s}{\partial u_{, i}} \dot{u}_{, i} \\
& +\frac{\partial s}{\partial q_{i, j}} \dot{q}_{i, j}+\frac{\partial J_{i}^{s}}{\partial u_{, j}} u_{, j i}+\frac{\partial J_{i}^{s}}{\partial \dot{q}_{j, k}} q_{j, k i} \geq 0
\end{aligned}
$$

To derive the restrictions imposed by $\sigma^{s}>0$, we follow the procedure proposed by Liu and Müller [25]; these authors introduce the constraints introduced by the evolution equations for $u$ and $q_{i}$ by means of Lagrange multipliers so that (18) takes the form

$$
\begin{aligned}
\sigma^{s} & -\Lambda_{u}\left(\dot{u}+q_{j, j}\right)-\Lambda_{i}\left(\dot{q}_{i}+\kappa u_{, i}+\frac{1}{\tau} q_{i}-\alpha_{1} q_{j} q_{j, i}-\alpha_{2} u_{, j} q_{j, i}\right. \\
& -\beta_{1} q_{i, j} q_{j}-\beta_{2} q_{i, j} u_{j}-\gamma_{1} q_{j, j} q_{i}-\gamma_{2} q_{j, j} u_{, i}-L_{1} q_{i, j j} \\
& \left.-L_{2} q_{j, j i}-L_{3} q_{j, i j}-L_{1}^{\prime} u_{, j} q_{i, j}-L_{2}^{\prime} u_{i} q_{j, j}-L_{3}^{\prime} q_{j, i} u_{, j}\right) \geq 0
\end{aligned}
$$

$\Lambda_{u}$ and $\Lambda_{i}$ are Lagrange multipliers $\left(\Lambda_{u}=\right.$ scalar, $\Lambda_{i}=i^{\text {th }}$ component of vector $\boldsymbol{\Lambda}$ to be identified at the end of the procedure. The consequences resulting from inequality (19) are established in the Appendix. The main results can be summarized as follows: i) $s$ is independent of $u_{, i}$ and $q, j$ so that one may write

$$
d s\left(u, q_{i}\right)=\frac{\partial s}{\partial u} d u+\frac{\partial s}{\partial q_{i}} d q_{i}
$$

As usual, we shall identify $\partial s / \partial u$ with the non-equilibrium temperature

$$
\frac{\partial s}{\partial u}=\theta^{-1}
$$


while $\partial s / \partial q_{i}$ is assumed to be linear in $q_{i}$ : as higher order terms in $q^{3}$ are omitted in the present analysis, one will simply take

$$
\frac{\partial s}{\partial q_{i}}=f(u) q_{i}
$$

where $f(u)$ is an undetermined function of $u$.

ii) the Lagrange multipliers are identified as

$$
\Lambda_{u}=\frac{\partial s}{\partial u}=\theta^{-1}, \quad \Lambda_{i}=\frac{\partial s}{\partial q_{i}}=f q_{i}
$$

iii) the entropy production takes the quadratic form

$$
\sigma^{s}=-\frac{f}{\tau} q_{i} q_{i}-L_{1} f q_{j, i} q_{j, i}-L_{2} f q_{j, j} q_{i, i}-L_{3} f q_{i, j} q_{j, i} \geq 0
$$

Expression (24) can also be given the form

$$
\sigma^{s}=-\frac{f}{\tau} q_{i} q_{i}-\left(L_{1}+L_{3}\right) f, q_{i, j}^{s y m} q_{i, j}^{s y m}-\left(L_{1}-L_{3}\right) f q_{i, j}^{s k e w} q_{i, j}^{s k e w}-L_{2} f q_{j, j} q_{i, i}
$$

where $q_{i, j}^{s y m}$ and $q_{i, j}^{\text {skew }}$ represent the symmetric and skew-symmetric parts of $q_{i, j}$. Positiveness of (25) implies that

$$
\frac{f}{\tau}<0, L_{2} f<0,\left(L_{1}+L_{3}\right) f<0,\left(L_{1}-L_{3}\right) f<0
$$

iv) an expression of the entropy flux compatible with the general results is

$$
J_{i}^{s}=T^{-1} q_{i}-L_{1} f q_{j} q_{j, i}-L_{2} f q_{i} q_{j, j}-L_{3} f q_{j} q_{i, j}
$$

with $T(u)$ the local equilibrium temperature.

v) The various coefficients appearing in the evolution equation (14) of $q_{i}$ are not independent but satisfy the following relations:

$$
L_{1} f^{\prime}=\beta_{2} f, \quad L_{2} f^{\prime}=\gamma_{2} f, \cdot L_{3} f^{\prime}=\alpha_{2} f
$$

where $f^{\prime}$ stands for $\partial f / \partial u$.

Supplementary information is provided from the equality of the mixed derivatives of the Gibbs equation

$$
d s=\theta^{-1} d u+f q_{i} d q_{i}
$$


Indeed, after integration of

$$
\frac{\partial \theta^{-1}}{\partial q_{i}}=f^{\prime} q_{i}
$$

it is found that

$$
\theta^{-1}\left(u, q^{2}\right)=\frac{1}{2} f^{\prime} q^{2}+T^{-1}(u)
$$

where $T(u)$ is the local equilibrium temperature as it corresponds to a vanishing value of the heat flux.

Moreover, convexity of entropy at fixed internal energy implies that $\partial^{2} s / \partial q_{i} \partial q_{i}<0$ from which follows that $f<0$. Therefore, in view of the inequalities (26), one has

$$
\tau>0, L_{1}>0, L_{2}>0, L_{1}>L_{3}>0,
$$

in agreement with earlier results of EIT and Guyer and Krumhansl's theory.

As a consequence of the above results, it is clear that there exists a strong correlation between the form of the evolution equation and the expressions of entropy and entropy flux.

\section{Particular situations}

It is our purpose to show that the above rather general results encompass well-known classical particular results. To this end, let us express the evolution equation (14) in terms of the temperature $\theta$ instead of $u$. One has

$$
\theta_{, i}^{-1}=\frac{\partial \theta^{-1}}{\partial u} u_{, i}+\frac{\partial \theta^{-1}}{\partial q_{j}} q_{j, i}
$$

The expression of $\partial \theta^{-1} / \partial u$ is directly obtained from (A.10) by taking into account the result (A.15):

$$
\frac{\partial \theta^{-1}}{\partial u}=\kappa f+\left(\gamma_{1} f\right)^{\prime} q^{2}
$$

Coupling this result with relation (30), it is found that

$$
\theta_{, i}^{-1}=\kappa f u_{, i}+\left(\gamma_{1} f\right)^{\prime} q^{2} u_{, i}+f^{\prime} q_{j} q_{j, i}
$$

from which follows that

$$
\kappa u_{, i}=-\frac{1}{f \theta^{2}} \theta_{, i}+\frac{f^{\prime}}{f} q_{j} q_{j, i}
$$

J. Non-Equilib Thermodyn. $\cdot 1998 \cdot$ Vol $23 \cdot$ No. 2 
wherein as before we have omitted the third order term in $q^{2} u, l$. Defining the heat conductivity $\lambda$ by

$$
\lambda=-\frac{\tau}{f \theta^{2}}
$$

and recalling that the results $\alpha_{1}=-\beta_{1}$ and $2 \gamma_{1}=f^{\prime} / f$ are compatible with the second law (see (A.22) and (A.23)), the evolution equation (14) for $q_{i}$ reads as

$$
\begin{aligned}
\dot{q}_{i}= & -\frac{\lambda}{\tau} \theta_{, i}-\frac{1}{\tau} q_{i}+\alpha_{1}\left(q_{j} q_{j, i}-q_{i, j} q_{j}\right)+\gamma_{1}\left(2 q_{j} q_{j, i}+q_{i} q_{j, j}\right) \\
& +\left(L_{3}^{\prime}+2 \gamma_{1} L_{3}\right) u_{, j} q_{j, i}+\left(L_{2}^{\prime}+2 \gamma_{1} L_{2}\right) u_{, i} q_{j, j}+\left(L_{1}^{\prime}+2 \gamma_{1} L_{1}\right) u_{, j} q_{i, j} \\
& +L_{1} q_{i, j j}+L_{2} q_{j, j i}+L_{3} q_{j, i j}
\end{aligned}
$$

this equation contains seven coefficients, namely $\tau, \lambda, \alpha_{1}, \gamma_{1}, L_{1}, L_{2}, L_{3}$, to be determined either by microscopic theories or by experimental measurements. At this point, it is interesting to consider the following two particular cases.

i) $f$ is a constant $\left(f^{\prime}=0\right)$.

By virtue of (28) and (A.18), the coefficients $\alpha_{2}, \beta_{2}, \gamma_{1}$ and $\gamma_{2}$ vanish and, according to (A.19), $\theta$ reduces to the local equilibrium temperature. Under these simplifications, the expressions of the entropy flux (27) and the heat transport equation (38) take respectively the following forms, if in addition $L_{1}, L_{2}$ and $L_{3}$ are assumed constant:

$$
\begin{aligned}
J_{i}^{s}= & T^{-1} q_{i}+\frac{\tau}{\lambda T^{2}}\left(L_{1} q_{j} q_{j, i}+L_{2} q_{i} q_{j, j}+L_{3} q_{j} q_{i, j}\right) \\
\dot{q}_{i}= & -\frac{\lambda}{\tau} T_{, i}-\frac{1}{\tau} q_{i}+\alpha_{1}\left(q_{j} q_{j, i}-q_{i, j} q_{j}\right) \\
& +L_{1} q_{i, j j}+L_{2} q_{j, j i}+L_{3} q_{j, i j} .
\end{aligned}
$$

By omitting in (40) the nonlinear contribution and putting $L_{1}=L_{2}=L_{3}$, one recovers Guyer-Krumhansl's equation, namely

$$
\dot{q}_{i}=-\frac{\lambda}{\tau} T_{, i}-\frac{1}{\tau} q_{i}+L_{1}\left(q_{i, j j}+2 q_{j, j i}\right)
$$

with the typical factor 2 in front of the term $q_{j, j i}$. The entropy flux corresponding to Guyer-Krumhansl's model is therefore

$$
J_{i}^{s}=T^{-1} q_{i}+\frac{L_{1} \tau}{\lambda T^{2}}\left(2 q_{j} q_{i, j}^{s y m}+q_{i} q_{j, j}\right)
$$

where $q_{i, j}^{s y m}$ is the symmetric part of tensor $q_{i, j}$. It is clear from (42) that GuyerKrumhansl equation is not compatible with the classical expression $T^{-1} q_{i}$ of the 
entropy flux. Note that on dimensional grounds one may write $\tau L_{i}=l_{i}^{2}(i=1,2,3)$ and therefore expressions (39)-(42) may be written in the form (3) and (4).

ii) $L_{1}=L_{2}=L_{3}=0$

By virtue of (28), one has $\alpha_{2}=\beta_{2}=\gamma_{2}=0$ and the evolution equation (38) is now given by

$$
\dot{q}_{i}=-\frac{\lambda}{\tau} \theta_{, i}-\frac{1}{\tau} q_{i}+\alpha_{1}\left(q_{j} q_{j, i}-q_{i, j} q_{j}\right)+\gamma_{1}\left(2 q_{j} q_{j, i}+q_{i} q_{j, j}\right),
$$

while the entropy flux is

$$
J_{i}^{s}=T^{-1} q_{i}
$$

This result provides a supplementary confirmation that the expression of the entropy flux is correlated to the presence of non-local terms of order two in the evolution equation for $q_{i}$.

A last remark is in form and concerns the comparison of the present results with Grad's theory, as expressed by equation (5).

Apparently, the form (5) of the entropy flux in Grad's theory is completely different from expressions (4) or (27) including the gradient of the heat flux. We will see here that in fact they are equivalent. Indeed, in the linear approximation, the evolution equations for the heat flux $q_{i}$ and the viscous pressure tensor $P_{i j}^{v}$ are (e.g. [14])

$$
\begin{aligned}
& \dot{q}_{i}=-\frac{1}{\tau_{1}} q_{i}-\frac{\lambda}{\tau_{1}} T_{, i}-\frac{k T}{m} P_{i j, j}^{v}, \\
& \dot{P}_{i j}^{v}=-\frac{1}{\tau_{2}} P_{i j}^{v}+2 \frac{\eta}{\tau_{2}} V_{i j}-\frac{4}{5} q_{i, j},
\end{aligned}
$$

wherein $V_{i j}$ is the velocity gradient tensor and $\eta$ the dynamic shear viscosity; by writing (45) and (46), it is assumed that the pressure tensor is traceless. Since in our problem, there is no global displacement of matter (zero velocity) and assuming that $\dot{P}_{i j}^{v}=0$, expression (46) becomes simply

$$
P_{i j}^{v}=-\frac{4}{5} \tau_{2} q_{i, j}
$$

Introducing (47) in (46) and in (5), one obtains respectively

$$
\begin{aligned}
& \dot{q}_{i}=-\frac{1}{\tau_{1}} q_{i}-\frac{\lambda}{\tau} T_{, i}+\frac{4}{5} \frac{k T}{m} \tau_{2} q_{i, j j}, \\
& J_{i}^{s}=T^{-1} q_{i}+\frac{8}{25 p T} \tau_{2} q_{i, j} q_{j},
\end{aligned}
$$

which are similar to the expressions derived in Section 3. Expression (48) allows us to express $L_{1}$ in terms of the relaxation time, namely $L_{1}=\frac{4}{5} \frac{\mathrm{kT}}{\mathrm{m}} \tau_{2}$. Furthermore, according to the kinetic theory, one has $\tau / \lambda=2 \mathrm{~m} / 5 \mathrm{kp}$ so that the coefficient $\tau^{-1} / \lambda T^{2}$ appearing in the entropy flux can be written as $8 \tau / 25 p T$ which is precisely the value of the coefficient of the non-classical term in (49). 
On the other hand, it follows from the information theory $[14,28,29]$ that the coefficients $\gamma_{0}$ and $\gamma_{1}$ in expression (6) are given by $\gamma_{0}=T^{-1}$ and $\gamma_{1}=-\frac{\tau}{\rho \lambda T^{2}} \mathbf{q}$ with $\rho$ the mass density. Comparing (6) with (42), it is seen that they are compatible by taking

$$
Q_{i j}=-\frac{\rho l_{1}^{2}}{\tau} 2 q_{i, j}^{s y m}
$$

\section{Conclusions}

Our objective was to formulate a general nonlinear and nonlocal heat transport equation well adapted to short-wave length and high frequencies processes. For simplicity, we have considered the problem of heat conduction in a rigid body; coupling between thermal and viscous effects will be examined in a future publication. Non-locality is introduced in the evolution equation of the heat flux $\mathbf{q}$ under the form of terms in $\nabla^{2} \mathbf{q}$, nonlinear contributions in $\mathbf{q} \cdot \nabla \mathbf{q}$ and $\nabla u \cdot \nabla \mathbf{q}$ are also taken into account. Expression (14) or (38) of the evolution equation generalizes that proposed by Guyer and Krumhansl some years ago; however these authors considered only the linear problem so that the present work can be viewed as a nonlinear extension of Guyer and Krumhansl formalism. It is also worth noting that the evolution equations (14) and (38) are obtained on completely different bases than Guyer and Krumhansl relation: the former are based on macroscopic thermodynamics while the latter is derived from Boltzmann equation.

An important conclusion drawn from the present study is that the expression of the entropy flux is directly correlated to that of the evolution equation and vice versa. This means that it would be not correct to make use of Guyer and Krumhansl's equation and to keep the classical form $T^{-1} q$ of the entropy flux. Indeed, it is shown that when non-locality is introduced, the entropy flux must contain additional terms in $\mathbf{q} \cdot \nabla \mathbf{q}$ whose coefficients are, apart a factor $f$, the same as these of the non-local contributions to the evolution equation. The factor $f$, a negative quantity, depends generally on the internal energy, or the temperature, and is related to the heat conductivity $\lambda$ and the relaxation time $\tau$ by $f=-\tau / \lambda \theta^{2}$. It is worth stressing that it is the same coefficient $f$ which appears in the EIT expression of the entropy which is of the form

$$
s\left(u, q_{i}\right)=s_{e q}(u)+f q_{i} q_{i},
$$

where $s_{e q}(u)$ is the local equilibrium entropy depending only on the internal energy.

A last remark concerns the temperature $\theta$. The latter is generally depending on the heat flux and can be cast in the form

$$
\theta^{-1}\left(u, q^{2}\right)=T^{-1}(u)+\frac{1}{2} f^{\prime} q^{2}
$$

where $T$ is the local equilibrium temperature; it is clear that $\theta$ reduces to its local equilibrium value only for $f=$ constant. 


\section{Acknowledgements}

This work was supported by EC Human Capital and Mobility Program, under contract ERB-CHRX-CT-92-0007, by the "InterUniversity Poles of Attraction Programme" (under contract IV-06), initiated by the Belgian State, Science Policy Programming and DGI CYT of the Spanish Government under grant PB 94-0718. Fruitful discussions with Prof. M. Grmela (University of Montréal) are acknowledged. D. Jou thanks Prof. Y. Katayama (Nikon University, Japan) for interesting discussions on nonlocal constitutive equations.

\section{Appendix}

Consequences resulting from the second law of thermodynamics

To examine the restrictions placed by inequality $\sigma^{s}\left(=\dot{s}+J_{i, i}\right) \geq 0$, we apply an elegant technique proposed by I-Shih-Liu and Müller [31] which consists in introducing the constraints imposed by the energy conservation law and the evolution equation of the heat flux via Lagrange multipliers. To be explicit, we shall formulate the second law of thermodynamics under the form

$$
\begin{aligned}
& \frac{\partial s}{\partial u} \dot{u}+\frac{\partial s}{\partial q_{i}} \dot{q}_{i}+\frac{\partial J_{i}^{s}}{\partial u} u_{, i}+\frac{\partial J_{i}^{s}}{\partial q_{j}} q_{j, i}+\frac{\partial s}{\partial u_{, i}} \dot{u}_{, i}+\frac{\partial s}{\partial q_{i, j}} \dot{q}_{i, j}+\frac{\partial J_{i}^{s}}{\partial u_{, j}} u_{, j i}+\frac{\partial J_{i}^{s}}{\partial q_{j, k}} q_{j, k i} \\
& \quad-\Lambda_{u}\left(\dot{u}+q_{j, i} \delta_{i j}\right)-\Lambda_{i}\left(\dot{q}_{i}+\kappa u_{, i}+\frac{1}{\tau} q_{i}-\alpha_{1} q_{j} q_{j, i}-\beta_{1} q_{j} q_{i, j j}\right. \\
& \quad-\gamma_{1} q_{i} q_{j, j}-\alpha_{2} u_{, j} q_{j, i}-\beta_{2} u_{, j} q_{i, j}-\gamma_{2} u_{, i} q_{j, j}-L_{1} q_{i, j j}-L_{2} q_{j, j i}-L_{3} q_{j, i j} \\
& \left.\quad-L_{1}^{\prime} u_{, j} q_{i, j}-L_{2}^{\prime} u_{, i} q_{k, k}-L_{3}^{\prime} u_{, j} q_{j, i}\right) \geq 0,
\end{aligned}
$$

wherein use is made of expression (18) of $\sigma^{s}$ and of evolution equations (7) and (14) for $u$ and $q_{i}$ respectively, $\Lambda_{u}$ and $\Lambda_{i}$ are the corresponding Lagrange multipliers. Rearranging the various terms in inequality (A.1), one obtains

$$
\begin{aligned}
& \left(\frac{\partial s}{\partial u}-\Lambda_{u}\right) \dot{u}+\left(\frac{\partial s}{\partial q_{i}}-\Lambda_{i}\right) \dot{q}_{i}+\frac{\partial s}{\partial u_{, i}} \dot{u}_{, i}+\frac{\partial s}{\partial q_{i, j}} \dot{q}_{i, j}+\frac{\partial J_{i}^{s}}{\partial u, j} u_{, j i} \\
& \quad+\left(\frac{\partial J_{i}^{s}}{\partial u}-\kappa \Lambda_{i}+L_{1}^{\prime} q_{j, i} \Lambda_{j}+L_{2}^{\prime} q_{j, j} \Lambda_{i}+L_{3}^{\prime} q_{i, j} \Lambda_{j}\right. \\
& \left.\quad+\alpha_{2} q_{i, j} \Lambda_{j}+\beta_{2} q_{j, i} \Lambda_{j}+\gamma_{2} q_{j, j} \Lambda_{i}\right) u_{, i} \\
& \quad+\left(\frac{\partial J_{i}^{s}}{\partial q_{j}}+\alpha_{1} \Lambda_{i} q_{j}+\beta_{1} q_{i} \Lambda_{j}+\gamma_{1} q_{k} \Lambda_{k} \delta_{i j}\right) q_{j, i} \\
& \quad+\left(\frac{\partial J_{i}^{s}}{\partial q_{j, k}}+L_{1} \Lambda_{j} \delta_{k i}+L_{2} \Lambda_{i} \delta_{j k}+L_{3} \Lambda_{k} \delta_{i j}\right) q_{j, k i} \\
& \quad-\Lambda_{u} q_{j, j}-\frac{1}{\tau} \Lambda_{i} q_{i} \geq 0 .
\end{aligned}
$$

J. Non-Equilib Thermodyn. $\cdot 1998 \cdot$ Vol $23 \cdot$ No. 2 
This inequality is linear in $\dot{u}, \dot{q}_{i}, \dot{u}_{, i}, \dot{q}_{i, j}$ and $u_{, j i}$, it is therefore inferred that positiveness of (A.2) demands that

$$
\frac{\partial s}{\partial u}=\Lambda_{u}, \quad \frac{\partial s}{\partial q_{i}}=\Lambda_{i}, \quad \frac{\partial s}{\partial u_{, i}}=0, \quad \frac{\partial s}{\partial q_{i, j}}=0, \quad \frac{\partial J_{i}^{s}}{\partial u_{, j}}=0 .
$$

As the remaining inequality is linear in $u_{, i}$ and $q_{j, k i}$, one has in addition

$$
\begin{aligned}
& \frac{\partial J_{i}^{s}}{\partial u}=\kappa \Lambda_{i}-L_{1}^{\prime} q_{j, i} \Lambda_{j}-L_{2}^{\prime} q_{j, j} \Lambda_{i}-L_{3}^{\prime} q_{i, j} \Lambda_{j}-\alpha_{2} q_{i, j} \Lambda_{j}-\beta_{2} q_{j, i} \Lambda_{j}-\gamma_{2} q_{j, j} \Lambda_{i} \\
& \frac{\partial J_{i}^{s}}{\partial q_{j, k}}=-L_{1} \Lambda_{j} \delta_{k i}-L_{2} \Lambda_{i} \delta_{j k}-L_{3} \Lambda_{k} \delta_{i j}
\end{aligned}
$$

and finally one is left with

$$
-\Lambda_{u} q_{j, j}-\frac{1}{\tau} q_{i} \Lambda_{i}+\left(\frac{\partial J_{i}^{s}}{\partial q_{j}}+\alpha_{1} \Lambda_{i} q_{j}+\beta_{1} q_{i} \Lambda_{j}+\gamma_{1} q_{k} \Lambda_{k} \delta_{i j}\right) q_{j, i} \geq 0
$$

Since $\partial s / \partial u$ is defined as the inverse of the temperature $\theta^{-1}\left(u, q^{2}\right)$ and recalling that $\partial s / \partial q_{i}$ is assumed to be linear in $q_{i}$, the Lagrange multipliers can be identified respectively as

$$
\Lambda_{u}=\theta^{-1}, \quad \Lambda_{i}=f(u) q_{i}
$$

A rather general expression for $J_{i}^{s}$ compatible with the result (A.5) is simply

$$
J_{i}^{s}=\phi\left(u, q^{2}\right) q_{i}-L_{1} f q_{j} q_{j, i}-L_{2} f q_{i} q_{j, j}-L_{3} f q_{j} q_{i, j},
$$

wherein $\phi\left(u, q^{2}\right)$ is, at this stage, an undefined function of $u$ and $q^{2}$. Making use of (A.7) and (A.8), inequality (A.6) reads as

$$
\begin{gathered}
q_{j, i}\left\{\left(-\theta^{-1}+\phi+\gamma_{1} f q^{2}\right) \delta_{i j}+\left[\left(\alpha_{1}+\beta_{1}\right) f+2 \frac{\partial \phi}{\partial q^{2}}\right] q_{i} q_{j}\right\} \\
-\frac{f}{\tau} q_{i} q_{i}-L_{1} f q_{j, i} q_{j, i}-L_{2} f q_{j, j} q_{i, i}-L_{3} f q_{i, j} q_{j, i} \geq 0 .
\end{gathered}
$$

Since the term between brackets is linear in $q_{i, j}$, positivity of (A.9) demands that

$$
\begin{aligned}
\theta^{-1} & =\phi+\gamma_{1} f q^{2}, \\
\frac{\partial \phi}{\partial q^{2}} & =-\frac{1}{2}\left(\alpha_{1}+\beta_{1}\right) f,
\end{aligned}
$$

so that (A.9) reduces to

$$
-\frac{f}{\tau} q_{i} q_{i}-L_{1} f q_{j, i} q_{j, i}-L_{2} f q_{j, j} q_{i, i}-L_{3} f q_{i, j} q_{j, i} \geq 0
$$


with, as direct consequence, that

$$
\frac{f}{\tau}<0, L_{2} f<0,\left(L_{1}+L_{3}\right) f<0,\left(L_{1}-L_{3}\right) f<0,
$$

It remains to examine the consequences resulting from the compatibility of (A.8) with (A.4). The derivation of (A.8) with respect to $u$ is given by

$$
\begin{aligned}
\frac{\partial J_{i}^{s}}{\partial u} & =\frac{\partial \phi}{\partial u} q_{i}-L_{1}^{\prime} f q_{j} q_{j, i}-L_{2}^{\prime} f q_{i} q_{j, j}-L_{3}^{\prime} f q_{j} q_{i, j} \\
& -L_{1} f^{\prime} q_{j} q_{j, i}-L_{2} f^{\prime} q_{i} q_{j, j}-L_{3} f^{\prime} q_{j} q_{i, j}
\end{aligned}
$$

and comparison with (A.4) leads to

$$
\begin{aligned}
& \frac{\partial \phi}{\partial u}=f \kappa, \\
& L_{1} f^{\prime}=\beta_{2} f, \quad L_{2} f^{\prime}=\gamma_{2} f, \quad L_{3} f^{\prime}=\alpha_{2} f .
\end{aligned}
$$

Using for $\theta^{-1}$ the result (31), it is found from (A.10) that a general expression for $\phi$ is

$$
\phi=T^{-1}(u)+\left(\frac{1}{2} f^{\prime}-\gamma_{1} f\right) q^{2}
$$

From the mixed derivatives of (A.10) with respect to $u$ and $q^{2}$, one obtains

$$
\frac{\partial^{2} \theta^{-1}}{\partial u \partial q^{2}}=\frac{\partial}{\partial q^{2}} \frac{\partial \phi}{\partial u}+\frac{\partial}{\partial u}\left(\gamma_{1} f\right)
$$

Since in virtue of (A.15), $\partial \phi / \partial u$ is independent of $q^{2}$, direct integration of (A.18) yields

$$
\frac{\partial \theta^{-1}}{\partial q^{2}}=\gamma_{1} f+C
$$

wherein $C$ is an integration constant. To determine $C$, recall that for $f=0$, the entropy $s(u)$ is only depending on $u$ as it results from (20) and (22). Consequently $\theta^{-1}(=\partial s / \partial u)$ will only be a function of $u$ and finally $C=0$. A new integration of (A.19) leads to

$$
\theta^{-1}=\gamma_{1} f q^{2}+T^{-1}(u)
$$

wherein $T$ is defined as the local equilibrium temperature as it corresponds to $q=0$. Supplementary consequences of the result (A.19) are the following. Substituting in (A.10), $\theta^{-1}$ by the result (A.19), it is directly seen that

$$
\phi=T^{-1}(u)
$$


and therefore, from (A.11),

$$
\alpha_{1}=-\beta_{1},
$$

and from (A.17)

$$
\gamma_{1}=\frac{1}{2} f^{\prime}
$$

\section{References}

[1] Tzou D. Y., An engineering assessment to the relaxation time in thermal wave propagation, Int. J. Heat Mass Transfer 36 (1993) 1845-1851.

[2] Sobolev S. L., Equations of transfer in nonlocal media, Int. J. Heat Mass Transfer 37 (1994) 2175-2182.

[3] Ferrer M., Jou D., Higher-order fluxes and the speed of thermal waves, Int. J. Heat Mass Transfer 34 (1991) 3055-3060.

[4] Ozisik M. N., Tzou D. Y., On the wave theory in heat conduction, J. Heat Transfer ASME 116 (1994) 526-535.

[5] Criado-Sancho M., Llebot J. E., On the admissible values of the heat flux in hyperbolic heat transport, Phys. Lett. A177 (1993) 323-326.

[6] Dreyer W., Struchtrup M., Heat pulse experiments revisited, Continuum Mech. Thermodyn. 5 (1993) 3-50.

[7] Joseph D. D., Preziosi L., Heat waves, Rev. Mod. Phys. 61 (1989) 41-73.

[8] Nettleton R. E., Sobolev S. L., Applications of extended thermodynamics to chemical, rheological and transport processes, part I, J. Non Equilib. Thermodyn. 20 (1995) 205229.

[9] Jou D., Casas-Vázquez J., Lebon G., Extended irreversible thermodynamics, Chap. 6, Springer, Berlin, 1993 (1st ed.), 1996 (2nd ed.).

[10] Lebon G., Jou D., Casas-Vázquez J., Extended irreversible thermodynamics: statements and prospects. in: Entropy and Entropy Generation: fundamentals and applications, pp 3754 Ed. J. S. Shiner, Kluwer, Dordrecht, 1996.

[11] Sieniutycz S., Conservation laws in variational thermohydrodynamics, Kluwer, Dordrecht, 1994.

[12] Lebon G., Torrisi M., Valenti A., A nonlocal thermodynamic analysis of second-sound propagation in crystalline dielectrics, J. Phys. Cond. Matter 7 (1994) 1461-1474.

[13] Nettleton R. E., The nonclassical entropy flux in extended thermodynamics, J. Chem. Phys. 97 (1992) 8815-8816.

[14] Maugin G. A., Nonlocal theories of gradient-like theories: a matter of convenience, Arch. Mechanics 31 (1979) 15-26.

[15] Aono O., Katayama Y., Thermodynamic forces and fluxes of a reacting system, Ann. Inst. Stat. Math., Tokyo, 40 (1994) 21-31.

[16] Lebon G., Grmela M., Weakly nonlocal heat conduction in rigid solids, Phys. Lett. A214 (1996) 184-188.

[17] Guyer R., Krumhansl H., Thermal conductivity, second-sound, and phonon hydrodynamics in nonmetallic crystals, Phys. Rev. 148 (1966) 778-788.

[18] Dedeurwaerdere T., Casas-Vázquez J., Jou D., Lebon G., Foundations and applications of a mesoscopic thermodynamic theory of fast phenomena, Phys. Rev. E53 (1996) 498-506.

[19] Müller I., The coldness, a universal function in thermoelastic bodies, Arch. Rat. Mech. Anal. 41 (1971) 319-332.

[20] Meixner J., Coldness and temperature, Arch. Rat. Mech. Anal. 57 (1972) 281-290.

[21] Grad H., Principles of the kinetic theory of gases, Handbuch der Physik, Springer, Berlin, 1959. 
[22] Dominguez-Cascante R., Jou D., Entropy flux and absolute temperature in extended irreversible thermodynamics, J. Non-Equilib. Thermodyn. 20 (1995) 263-273.

[23] Nettleton R. E., The Gibbs equation from maximum entropy, J. Chem. Phys. 93 (1990) 8247-8253.

[24] Cattaneo C., Sur une forme de l'équation de la chaleur éliminant le paradoxe d'une propagation instantanée, Comptes rendus Ac.Sc. Paris, 247 (1958) 431-433.

[25] Shih-Liu I., Müller I., Extended thermodynamics of classical and degenerate ideal gases, Arch. Rat. Mech. Anal. 83 (1983) 285-332.

Paper received: 1998-2-19

Paper accepted: 1998-4-2

G. Lebon

Liège University

Institute of Physics B5

Sart Tilman

B-4000 Liège 1

Belgium

D. Jou and J. Casas-Vázquez

Autonomous University of Barcelona

Department of Statistical Physics

E-08193 Bellaterra (Barcelona)

Spain

W. Muschik

Technical University Berlin

Institute for Theoretical Physics

Hardenbergstrasse 36

10623 Berlin

Germany 